\title{
Multiallelic rare variants support an oligogenic origin of sudden cardiac
}

\section{death in the young}

Hager JAOUADI ${ }^{1}$, Yosra BOUYACOUB ${ }^{1}$, Sonia CHABRAK ${ }^{2,3}$, Lilia KRAOUA ${ }^{4}$, Amira ZAROUI $^{3}$, Sahar ELOUEJ ${ }^{5}$, Majdi NAGARA ${ }^{1}$, Hamza DALLALI ${ }^{1}$, Valérie DELAGUE ${ }^{5}$, Nicolas LEVY ${ }^{5}$, Rym BENKHALIFA ${ }^{6}$, Rachid MECHMECHE ${ }^{2,3}$, Stéphane ZAFFRAN ${ }^{5}$, Sonia ABDELHAK ${ }^{1}$.

${ }^{1}$ Biomedical Genomics and Oncogenetics Laboratory LR16IPT05, Institut Pasteur de Tunis, Université Tunis El Manar, Tunis, Tunisia.

${ }^{2}$ Department of Cardiology, La Rabta Hospital, Tunis, Tunisia.

${ }^{3}$ Faculty of Medicine of Tunis, Université Tunis El Manar, Tunis, Tunisia.

${ }^{4}$ Department of Congenital and Hereditary Diseases, Charles Nicolle Hospital, Tunis, Tunisia.

${ }^{5}$ Aix Marseille University, INSERM, Marseille Medical Genetics, U1251, Marseille, France.

${ }^{6}$ Venoms and Therapeutic Biomolecules Laboratory LR16IPT08, Institut Pasteur de Tunis, Tunisia.

\section{Corresponding Author:}

Hager Jaouadi, $\mathrm{PhD}$

Biomedical Genomics and Oncogenetics Laboratory LR16IPT05

Institut Pasteur de Tunis

13, Place Pasteur, BP74-1002 Tunis, belvédère, Tunisie

Tel: +21671843755

E-mail: jaouadihager@gmail.com

ORCID: 0000-0002-6806-0072

Conflicts of interest: The authors declare that they have no conflict of interest. 


\begin{abstract}
Unexplained sudden death in the young is cardiovascular in most cases. Structural and conduction defects in cardiac-related genes can conspire to underlie sudden cardiac death. Here we report on a clinical investigation and an extensive genetic assessment of a Tunisian family with sudden cardiac death in young members. In order to identify the family-genetic basis of sudden cardiac death, we performed Whole Exome Sequencing (WES), read depth copy-number-variation (CNV) screening and segregation analysis. We identify 6 ultra-rare heterozygous variants in $O B S C N, R Y R 2, D S C 2, A K A P 9, C A C N A 1 C$ and $R B M 20$ genes and one homozygous splicing variant in TECRL gene consistent with an oligogenic model of inheritance. CNV analysis did not reveal any causative CNV. Overall, our results are highly suggestive for a cumulative effect of several variants as disease causation and to account for a greater disease severity among offspring. Our study further confirms the complexity of the inheritance of sudden cardiac death and highlights the utility of family-based WES in the identification of family specific mutations within different cardiac genes pathways.
\end{abstract}

Keywords: calcium mishandling; oligogenic inheritance; sudden unexplained cardiac death; TECRL gene; whole exome sequencing. 


\section{Introduction}

Sudden unexplained death in the young is defined as an unexplained sudden death occurring in an individual older than 1 year of age (Priori et al. 2013; Brion et al. 2015). The cause of death remains unexplained after a thorough investigation including performance of autopsy, review of the circumstances of death and clinical history (Wilhelm et al. 2015; Neubauer et al. 2017). A major proportion of sudden unexplained deaths are considered to be caused by inherited cardiac diseases (Hata et al. 2016). Sudden death may be the first manifestation of these diseases and initial phenotypic alterations may not be visible at autopsy or it can be considered unspecific or within the normal range (Christiansen et al. 2016). Contrary to patients aged over 40 years, whose coronary artery disease is the main trigger of sudden cardiac death, the implication of genetic factors in young cases is the major cause of sudden cardiac death (Brion et al. 2015).

Cardiologic and molecular genetic evaluation of first-degree relatives of young individuals experiencing sudden cardiac death is recommended (Behr et al. 2003, 2008; van der Werf et al. 2010). Testing yields from large sudden unexplained death cohort studies varied from 10 to $30 \%$ (Bagnall et al. 2014; Lahrouchi et al. 2017). This low yield of genetic testing may be explained by the complex genetic etiology of this cardiac disorder and the implication of novel genes, not yet identified.

Several studies suggest that alterations of the $\mathrm{Ca}^{2+}$ homeostasis in the sarcomere and electric dysfunction as a new pathophysiological mechanism underlying sudden cardiac death (Brion et al. 2012, 2014). 
Cardiac arrhythmias syndromes are mostly inherited in an autosomal dominant manner but may exhibit incomplete penetrance, a high variable expressivity and phenotypic overlap. This fact is probably due to a combination of genetic variants and the additive effect of multiple independent mutations may increase the severity of the phenotype (Coll et al. 2017). Here, we report the results of clinical and genetic investigation of a Tunisian family with high incidence of sudden cardiac death in young members.

\section{Material and methods}

\section{Whole Exome Sequencing (WES)}

Peripheral blood sample was collected after obtaining the written informed consent from all family members included in this study or their guardians. Genomic DNA was extracted by standard techniques from the codified samples.

Whole exome sequencing was performed for both parents (II:1 and II:2), the deceased child (III:4) and the youngest child (III:5) (Figure 1), using the NimbleGen SeqCap EZ MedExome kit (total design size $47 \mathrm{Mb}$ ) according to the manufacturer's protocol (Roche Sequencing Solutions, Madison, USA). Paired-end 150-bp reads from the DNA libraries were sequenced using Illumina NextSeq 500 platform (Illumina, San Diego, CA, USA).

Raw data were mapped to the built of the human genome (hg19) by using BWA 0.7.5. Variant calling and annotation were performed using GATK and ANNOVAR, respectively. Variant annotation process and exome analysis were performed with VarAFT software, version 2.06 (http://varaft.eu/).

\section{Variant filtering strategy}


Several filter settings were applied to pinpoint putatively causal variants according to both autosomal recessive and autosomal dominant pattern of inheritance. In order to reduce the significant number of variants, we first excluded variants with a Minor Allele Frequency $(\mathrm{MAF})>1 \%$ in ExAC database (http://exac.broadinstitute.org/). Then, we removed all noncoding and synonymous variants. Subsequently, the variant list (including rare, nonsense, missense, splice site variants and Indels) was filtered according to the following in silico prediction algorithms: Sorting Tolerant From Intolerant (http://sift.jcvi.org/), PolyPhen-2 (Polymorphism Phenotyping v2; http://genetics.bwh.harvard.edu/pph2/) and UMD Predictor (http://umd-predictor.eu/).

In a second step, to select potential pathogenic and clinically relevant variants, we performed a targeted gene analysis using a large list containing 198 known cardiomyopathy-associated genes (including cardiomyopathies, arrhythmias and channelopathies-associated genes) and genes known to cause metabolic disease and/or genetic syndromes (Suppl.Table1).

More accurately, for the autosomal recessive model, a pedigree based analysis was performed, considering (i) both III :4 and III :5 as affected members (candidate variant present in homozygous state in III :4 and III :5) and (ii) III :4 as affected member and III :5 as unaffected (candidate variant present in homozygous state in III :4 and in heterozygous state or absent in III :5). Similarly, we applied the same strategy of analysis assuming an autosomal dominant pattern of inheritance.

\section{Sanger validation}

In order to validate whole exome sequencing results, all the putative causal variants prioritized were confirmed by Sanger sequencing. 
WES variants filtering and prioritization strategy is summarized in Figure 2.

\section{CNV calling}

Copy number variations were called from WES data using ExomeDepth (version 1.1.10, https://CRAN.R-project.org/package=ExomeDepth). ExomeDepth has been reported to be better than most methods of exome-based read depth CNV calls (Plagnol et al. 2012; Tan et al. 2014; Lopes et al. 2015).

\section{CNV annotation and prioritization}

AnnotSV (https://lbgi.fr/AnnotSV/) was used to annotate CNV by incorporating CNV data from OMIM ( $\quad$ https://www.omim.org/), 1000Genomes Project (http://www.internationalgenome.org/), ExAC, DGV (http://dgv.tcag.ca/dgv/app/home) and DECIPHER (https://decipher.sanger.ac.uk/). Thus, to filter out common CNV we selected the DGV variants overlapping at least $70 \%$ of $\mathrm{CNV}$ identified in our family members. Moreover, AnnotSV generates for each CNV one annotation based on the full length $\mathrm{CNV}$ and one annotation for each gene within the $\mathrm{CNV}$, which aid to detect the potentially causative CNV. In addition to these annotations, AnnotSV provides a classification delineated by the American College of Medical Genetics and Genomics (ACMG). Hence, for our CNV analysis and prioritization we first performed a gene-based search to determine if genes implicated in the cardiovascular system are included in the identified CNV. Then, we focused on CNV classified as 'likely pathogenic' and 'pathogenic' according to the ACMG guidelines and CNV affecting genes in cardiac-related pathways.

The genetic study design of the family is summarized in Figure 3.

\section{Results}




\section{Family members' clinical data}

Clinical findings and personal history of family members are described below. Family pedigree is shown in Figure 1.

II: 2 (proband). The proband is 42 years-old, she had a history of 2 syncopes in a vasovagal context at the age of 28 and 29 years. A 12-lead ECG did not show any anomalies. Flecainide test was performed three times, it was positive once. Echocardiography revealed a slight dilation of right ventricle. After the sudden death of her two children (III:1 and III:2) she received an implantable cardioverter defibrillator (ICD) at the age of 29 years-old. Since the implantation, the ICD has never shocked; she is treated with CoAprovel for her hypertension.

III:1 he was asymptomatic until the age of 8 years-old and 2 months when he deceased suddenly while playing.

III:2 she died suddenly at the same age of her brother's death ( 8 years-old and 2 months) while she was walking. She was asymptomatic.

III:3 is the fourth proband's pregnancy. Pregnancy was interrupted at 32 weeks by spontaneous miscarriage. Autopsy of the fetus was performed and revealed a structurally normal heart.

III: 4 is the third deceased child. She died suddenly at the age of 8 years-old and 4 months when she was at school. Her first cardiac examination was without abnormalities except a pulmonary valvular stenosis of very low gradient. Flecainide test was performed twice (at 3 and 5 years-old) and was negative. Rhythm monitoring did not show any significant cardiac arrhythmias. Doppler echocardiography performed at 3 years-old revealed left ventricular dilation without hypertrophy with a well-preserved function (end-diastolic diameter $=31 \mathrm{~mm}$ 
and end-systolic diameter $=17 \mathrm{~mm}$ ). Neither aortic insufficiency nor valvular aortic stenosis were observed. She had syncope at the age of 5 years-old upon waking up in the morning. Last cardiac examination was at the age of 6 years-old and she was asymptomatic at that time.

III:5 is the youngest proband's child, although she was asymptomatic and no conclusive diagnosis was made, a decision of implantation of an implantable cardioverter defibrillator was taken owing to the sudden deaths of her three siblings. Her 12-lead ECG was normal except signs of short PR interval associated to early repolarization. Holter monitoring was normal except 2 ventricular extra-systoles that occurred in the morning. Magnetic resonance imaging revealed a slight ventricular dilation.

II: 1 is the proband's husband. They are first cousins. His cardiac examination was normal. He has no personal history of syncope or any other heart discomfort.

\section{Family history and extended cardiac evaluation}

I:2 is the proband's father, he is 63 years-old. His medical history was unremarkable. A 12lead ECG did not show any abnormalities.

Cardiac examination of other family members showed that the maternal uncle of the proband (I:9), followed for several years for ventricular excitability disorder, had arrhythmogenic cardiomyopathy as well as his son (II:8). Family investigation revealed a high number of sudden deaths: two maternal uncles died suddenly at 24 and 25 years-old (I:5 and I:6) and one proband's brother died at a very early age (II:4). Unfortunately, no DNA of this branch of the family was available.

\section{Genetic investigation of the family}




\section{SCN5A sequencing}

Taking into account the clinical data of the proband including mainly: 2 episodes of syncope, positive response to the flecainide test and the sudden death of her 2 children, the SCN5A gene was first sequenced but no causative mutation was found. Therefore, a whole exome sequencing was performed for the proband (II:2), the deceased child (III:4), the youngest child (III:5) and their father (II:1) in a research context in order to identify the genetic basis underlying the sudden death in the family.

\section{Whole exome sequencing}

The average coverage of all targeted exome was $95 \%$ at $>=20 \mathrm{X}$ and $92 \%$ at $>=30 \mathrm{X}$. The use of whole exome sequencing enabled us to perform a targeted analysis by focusing on 198 cardiac-related genes. Using the in-house VarAFT software, both recessive and dominant models of inheritance have been performed.

In total, 7 missense variants and one splice-site variant were identified as pathogenic and likely causative (Table 1). 
Table 1: List of variants identified in this study.

\begin{tabular}{|c|c|c|c|c|c|c|c|c|c|c|c|c|c|c|}
\hline Gene & $\begin{array}{c}\text { OMIM } \\
\text { number/phenotype }\end{array}$ & cDNA change & $\begin{array}{l}\text { Protein } \\
\text { change }\end{array}$ & $\begin{array}{l}\text { Geno- } \\
\text { type }\end{array}$ & Inheritance & rs ID & $\begin{array}{c}\mathrm{AF} \\
\text { ExAC }\end{array}$ & $\begin{array}{c}\text { AC } \\
\text { gnom } \\
\text { AD }\end{array}$ & $\begin{array}{c}\mathrm{AF} \\
\text { gnomAD }\end{array}$ & $\begin{array}{c}\text { AF } \\
\text { GME } \\
\text { Variome } \\
\end{array}$ & SIFT & PolyPhen & $\begin{array}{l}\text { UMD } \\
\text { score }\end{array}$ & $\begin{array}{l}\text { UMD } \\
\text { prediction }\end{array}$ \\
\hline$O B S C N$ & $\begin{array}{c}\text { 608616/DCM/ } \\
\text { HCM }\end{array}$ & c. $20563 \mathrm{C}>\mathrm{A}$ & p.R6855S & HTZ & MI & NA & NA & NA & NA & NA & $\mathrm{D}$ & $\mathrm{D}$ & 96 & Pathogenic \\
\hline$D S C 2$ & 125645/ARVD11 & c. $2603 \mathrm{C}>\mathrm{T}$ & p.S868F & HTZ & MI & rs141873745 & $5.769^{e-5}$ & 13 & $4.694^{e-5}$ & 0.001 & $\mathrm{D}$ & $\mathrm{D}$ & 93 & Pathogenic \\
\hline$V C L$ & $\begin{array}{c}\text { 193065//DCM/ } \\
\text { HCM }\end{array}$ & c. $2046 \mathrm{~A}>\mathrm{T}$ & p.L682F & HTZ & MI & rs565398652 & $6.589^{\mathrm{e}-5}$ & 17 & $6.132^{\mathrm{e}-5}$ & NA & $\mathrm{T}$ & $\mathrm{D}$ & 75 & Pathogenic \\
\hline$R Y R 2$ & $\begin{array}{c}\text { 180902/ARVD2/ } \\
\text { CPVT1 }\end{array}$ & c. $3660 \mathrm{~T}>\mathrm{A}$ & p.D1220E & HTZ & MI & rs 1019112544 & NA & NA & NA & NA & $\mathrm{D}$ & B & 69 & $\begin{array}{c}\text { Probably } \\
\text { pathogenic }\end{array}$ \\
\hline$A K A P 9$ & 604001/LQTS11 & c. $4826 \mathrm{G}>\mathrm{A}$ & p.R1609K & HTZ & MI & rs148146011 & 0.0004 & 133 & 0.0048 & 0.006 & $\mathrm{~T}$ & B & 69 & $\begin{array}{c}\text { Probably } \\
\text { pathogenic }\end{array}$ \\
\hline CACNAIC & $\begin{array}{c}\text { 114205/ BrS 3/ } \\
\text { LQTS 8/ Timothy S }\end{array}$ & c. $5383 \mathrm{G}>\mathrm{A}$ & p.G1795R & HTZ & PI & rs111298509 & 0.0057 & 1295 & 0.005 & 0.003 & $\mathrm{~T}$ & $\mathrm{D}$ & 100 & Pathogenic \\
\hline$R B M 20$ & 613171/DCM & c. $1364 \mathrm{C}>\mathrm{T}$ & p.S455L & HTZ & PI & rs189569984 & 0.0048 & 954 & 0.009 & 0.001 & $\mathrm{D}$ & $\mathrm{D}$ & 66 & $\begin{array}{c}\text { Probably } \\
\text { pathogenic }\end{array}$ \\
\hline TECRL & 614021/CPVT 3 & c. $331+1 \mathrm{G}>\mathrm{A}$ & $\begin{array}{l}\text { IVS3DS, } \\
\text { G-A, }+1\end{array}$ & $\mathrm{HMZ}^{*}$ & MI and PI & rs1057517699 & NA & NA & NA & NA & NA & NA & NA & NA \\
\hline
\end{tabular}

OMIM: Online Mendelian Inheritance in Man; DCM: Dilated cardiomyopathy; HCM: Hypertrophic cardiomyopathy; ARVD: Arrythmogenic Right Ventricular Dysplasia; CPVT: Catecholaminergic Polymorphic Ventricular Tachycardia; LQTS: Long QT Syndrome; BrS: Brugada syndrome; HTZ: Heterozygous; HMZ*: Homozygous in III:4 member; MI: maternally inherited; PI: paternally inherited; The Genome Aggregation Database (gnomAD): 123,136 exomes and 15,496 genomes; The Greater Middle East (GME) Variome: 2,497 exomes; AC: Allele count, AF: Allele frequency; D: Deleterious; T: Tolerated; B: Benign. 
Variants were evaluated one by one in concert with personal and familial history in order to determine the most likely causative variants. Moreover, to help in the interpretation process we assessed the functional impact of the identified variants using the Combined Annotation Dependent Depletion (CADD) C-scores. The score cutoff on deleteriousness used $>15$ (http://cadd.gs.washington.edu) (Table 2).

Table 2: Prediction of variant deleteriousness based on CADD-scores.

\begin{tabular}{|l|l|}
\hline Variant & CADD Phred \\
\hline OBSCN: c.20563C >A; p.R6855S & 35 \\
DSC2: c.2603C > T; p.S868F & 31 \\
VCL: c.2046A $>$ T; p.L682F & 23.3 \\
RBM20: c.1364C $>$ T; p.S455L & 23.3 \\
RYR2: c.3660T>A; p.D1220E & 23.2 \\
AKAP9: c.4826G >A; p.R1609K & 22.8 \\
CACNA1C $:$ c.5383G $>$ A; p.G1795R & 17.26 \\
\hline
\end{tabular}

As CADD and other prediction tools mentioned in Table 1 do not predict splice site variants, we used Human Splicing Finder "HSF" (http://www.umd.be/HSF/HSF.shtml) to better understand the effect of the TECRL; c. $331+1 \mathrm{G}>\mathrm{A}$ variant on the mRNA processing. Two signals have been predicted by $\operatorname{HSF}(i)$ a new donor site: the TECRL; c. $331+1 \mathrm{G}>\mathrm{A}$ variant is predicted to activate an intronic cryptic donor site leading to a potential splicing alteration (ii) a broken WT donor site consisting in the alteration of the WT donor site and most probably affecting splicing. Moreover, the TECRL; c. $331+1 \mathrm{G}>\mathrm{A}$ variant is predicted as pathogenic according to ClinVar (https://www.ncbi.nlm.nih.gov/clinvar/variation/372283/).

Since familial segregation is one of the strongest evidence for causality, we checked the state of proband's father (I:2), whose cardiac examination did not show any abnormalities and he did not have a remarkable medical history. 
The proband's father (I:2) was negative for all the prioritized variants, except the $V C L$; p.L682F variant. Accordingly, it will be considered as not causative of cardiac phenotypes in the present family.

\section{CNV analysis}

WES-read depth CNV analysis was performed in order to unravel the contribution of potential causal CNV to the genetic etiology of sudden cardiac death in the family. Thus, rare and clinically relevant $\mathrm{CNV}$ consisting with the family phenotype were searched. After filtering process two $\mathrm{CNV}$ harboring protein coding genes related to the cardiovascular system were identified (Suppl.Table 2).

The first CNV is a duplication encompassing 2 exons of PIEZO1 gene. Piezo1 is a calcium permeable mechanosensitive channel which induces mechanically activated currents in numerous cell types including cardiomyocytes (Coste et al. 2010; Wong et al. 2018). Moreover, recent studies suggest a pivotal role for Piezo1 in pathophysiological phenotypes such as, pulmonary arterial hypertension and right heart remodeling (Rode et al. 2017).

The second CNV duplication contained the ABCC6 gene which is associated to the generalized arterial calcification of infancy (MIM number 614473).

Both identified CNV among III:4 and III:5 members are biparentally inherited and inconsistent with the family phenotype (Suppl.Table 2). Consequently, our CNV analysis did not detect causative CNV.

\section{Discussion}


A significant number of sudden death cases remain unexplained despite a detailed postmortem investigation (Kauferstein et al. 2013) and more than $80 \%$ of all sudden death cases are of cardiac origin (Adabag et al. 2010). Previous studies showed that above $40 \%$ of sudden cardiac death victims did not present overt cardiac anomalies after autopsy (Priori et al. 1992; Winkel et al. 2011). Moreover, lethal arrhythmia represents the most plausible cause of death (Neubauer et al. 2017).

Approximately $40 \%$ of sudden unexplained death in the young cases are caused by both structural and non-structural heart abnormalities (Basso et al. 2010; Bagnall et al. 2014). Indeed, the mechanism underlying sudden cardiac death is ventricular arrhythmias which is more likely associated with a dysfunction of the electrical activity in the heart, a key factor of the cardiac rhythmicity (Maron 2003). Mutations in genes encoding cardiac ion channels and their related proteins leading to a gain or a loss of function of one or more ionic currents alters the balance between the depolarizing and repolarizing forces during the ventricular action potential (Brion et al. 2015). Thus, in our analysis we considered genes encoding ion channels as well as structural proteins due to cardiac phenotypes continuum and the clinical and genetic overlap (Noorman et al. 2013; Agullo-Pascual et al. 2014; Sarquella-Brugada et al. 2016).

In the present study, we report on a Tunisian family with a marked history of sudden unexplained cardiac death. Genetic investigation of $S C N 5 A$ gene ruled-out the presence of SCN5A-related disorders. Hence, family-based whole exome sequencing was performed and 8 ultra-rare pathogenic variants were identified consistent with an oligogenic model of inheritance (Table1).

The proband and her offspring (II:2, III:4 and III:5) share 4 likely pathogenic heterozygous variants in $O B S C N, D S C 2, R Y R 2$ and $A K A P 9$ genes. The children (III:4 AND III:5) carry, in 
addition to the maternally inherited variants, two other heterozygous variants inherited from their father (II:1) in RBM20 and CACNA1C genes. The TECRL; c.331+1G>A variant is found to be homozygous in the deceased child (III:4) and heterozygous in both parents (II:1, II:2) and the child (III:5) (Figure 1). We hypothesize that the combination of all these likely pathogenic variants could explain the phenotype and the occurrence of sudden cardiac death among the children. Of note, the individual III:5 had an implantable cardioverter defibrillator (ICD) at the age of 8 years-old in order to prevent lethal arrhythmias. This decision was made after the death of all her siblings and before the genetic investigation by WES was performed.

The $O B S C N$; c.20563C $>\mathrm{A}$; p.R6855S variant is absent in all public databases as well as in our in-house database of Tunisian exomes. The OBSCN gene has been recently associated to different subsets of cardiomyopathies (Arimura et al. 2007; Marston et al. 2015; Marston 2017a). Martson et al. reported that $O B S C N$ variants may act in concert with variants in the same or other genes to initiate a cardiac pathology (Marston et al. 2015; Marston 2017a). Moreover, mutations in sarcomeric genes have been related to sudden cardiac death in the absence of conventional risk factors. The disruption of sarcomeric activity could alter intracellular calcium homeostasis and be responsible for arrhythmogenesis (Brion et al. 2012). Interestingly, the $O B S C N$ gene interacts directly with sarcomeric proteins and $\mathrm{Ca}^{2+}$ handling proteins of the sarcoplasmic reticulum (Arimura et al. 2007; Marston 2017b). According to the scoring of combined annotation dependent depletion (CADD), the $O B S C N$ c.20563C $>$ A; p.R6855S variant, identified in our study, has the highest score of deleteriousness (Table 2). Considering all these findings and given the rarity and novelty of this variant, we hypothesize that it may contribute to the severity of the phenotype. 
The RYR2; c.3660T>A; p.D1220E variant has not been previously reported in the literature. In addition, the variant is absent in all population databases. We also queried our in-house Tunisian database and the variant is not present. Recently, the RYR2; c.3660T>A; p.D1220E was submitted by Trans-Omics for Precision Medicine (TOPMed) in dbSNP (https://www.ncbi.nlm.nih.gov/SNP/snp_ref.cgi?rs=rs1019112544 ). The RYR2 gene encodes a ryanodine receptor type 2 expressed in sarcoplasmic reticulum of the cardiac muscle. It mediates $\mathrm{Ca}^{2+}$ release from the sarcoplasmic reticulum into the cytoplasm and plays a crucial role in triggering cardiac muscle contraction. The dysregulation of $R Y R 2$ leads to potentially lethal cardiac arrhythmias (Huang et al. 2014). Indeed, RYR2 mutations lead to spontaneous diastolic $\mathrm{Ca}^{2+}$ release that activates the $\mathrm{Na}^{+} / \mathrm{Ca}^{2+}$ antiporter by increasing $\mathrm{Ca}^{2+}$ levels in the cytosol (Bezzina et al. 2015).

Interestingly, the models of smooth and cardiac muscle excitation-contraction coupling assume that $C A C N A 1 C$ and $R Y R 2$ affect the activity of each other only through changes in $\mathrm{Ca}^{2+}$ at the calcium release unit (Franzini-Armstrong 2004). A mode of interaction and communication between RYR2 and CACNA1C via Homer 1 scaffolding protein has been reported, this has an important implications for the regulation of smooth and possibly cardiac muscle excitation-contraction coupling (Huang et al. 2007).

The activation of the L-type calcium channel $\mathrm{Ca}_{\mathrm{v}} 1.2$ encoded by the CACNA1C gene increases the $\mathrm{Ca}^{2+}$ level that triggers the calcium release in cardiomyocytes (Neubauer et al. 2017). A molecular and functional study in 82 sudden unexplained death in the young cases provides evidence that $C A C N A 1 C$ rare variants may contribute to the pathogenic basis of some autopsy-negative sudden unexplained death in the young cases through either gain or loss of function mechanism (Sutphin et al. 2016). 
Furthermore, several studies have investigated the relation between genes of the desmosome and components of cardiac electrical activity (Delmar 2012; Noorman et al. 2013; Cerrone and Delmar 2014). Previous cases of sudden cardiac death carrying multiple mutations in both structural and non-structural genes have been reported (Sarquella-Brugada et al. 2016). Hence, the combination of variants in genes implicated in the $\mathrm{Ca}^{2+}$ release mechanisms such as RYR2, CACNAIC and the desmosomal gene DSC2 might lead to the occurrence of arrhythmias events in our cases.

The TECRL; c.331+1G>A variant has been reported once in a Sudanese family with CPVT (Devalla et al. 2016). In this same study, Devalla et al., reported two other families with overlapping clinical features of long QT syndrome and CPVT carrying mutations in TECRL gene. The TECRL gene encoded the trans-2,3-enoyl-CoA reductase-like protein which is an endoplasmic reticulum protein predominantly expressed in the heart. More recently, a second study confirmed the association of heterozygous compound mutations in the TECRL gene with CPVT (Xie et al. 2019). Of note, in the present family, no additional variants in the TECRL gene were found. Thus, we reported the second family in the literature harboring the c.331+1G>A variant in TECRL. However, the medical history of the family and the latest cardiac evaluation of the mother (II:2) and her daughter (III:5) did not show any recognized symptoms of long QT syndrome or CPVT.

We hypothesize that TECRL gene could be associated to other cardiac disorders and/or contribute to the high risk of severe arrhythmias. Furthermore, heterozygote carriers of the TECRL variant may present a variable expressivity of the cardiac phenotype or be asymptomatic. Indeed, the assessment of risk status of heterozygous carriers of TECRL 
mutations will require the identification of a larger number of cases and genotype/phenotype correlations.

On the other hand, variants in $A K A P 9$ gene have been associated with cardiac event risk as well as disease severity (de Villiers et al. 2014). Recently, Neubauer et al. (2018) investigated a cohort of 34 sudden unexplained death victims by focusing on candidate genes involved in cardiomyopathies and channelopathies and identified a heterozygous missense variant in $A K A P 9$ in one case (Neubauer et al. 2018).

Additionally, mutations in RBM20 have been linked to aggressive form of familial dilated cardiomyopathy (Wells et al. 2013; van den Hoogenhof et al. 2018). A recent study revealed that $\mathrm{Ca}^{2+}$ mishandling caused by complete and heterozygous $R B M 20$ mutations underlies the high risk of malignant arrhythmias. Indeed, functional studies showed splicing abnormalities in $\mathrm{Ca}^{2+}$ and ion handling channels such as $R Y R 2$ after loss of $R B M 20$ in human, rat and mouse (van den Hoogenhof et al. 2018).

Most likely, variants in $R B M 20$ and $A K A P 9$ genes may modify the clinical expression of the disease but not directly associated with the occurrence of a life-threatening rhythmic event among the children of the present family.

In the present study, the inheritance pattern of the missense variants and the TECRL splicing variant identified is consistent with an oligogenic model of inheritance. Oligogenic inheritance may be associated with more severe symptoms such as acceleration of the disease progression and life-threatening arrhythmias (Bezzina et al. 2015; Li et al. 2017).

Although some cardiac disorders are clearly mendelian with a strong monogenic cause, the pattern of inheritance of numerous heritable cardiac diseases still unclear. Several studies 
challenged the concept of "one gene one disease" and a complex pattern of inheritance has been proposed. Bezzina et al. supported the notion that a continuum of complexity of genetic factors exists. Therefore, other models of inheritance have been advanced such as: "near mendelian" inheritance where a strong genetic factor is modulated by additional genetic variants whereas other diseases will be oligogenic (Bezzina et al. 2015).

It has been reported that a subset of hypertrophic cardiomyopathy might be oligogenic caused by several pathogenic variants in genes implicated in familial hypertrophic cardiomyopathy and their aggregation is associated with the severity of the phenotype ( $\mathrm{Li}$ et al. 2017). In the same context, Gifford et al. (2018) showed that a human congenital heart defect, the left ventricular non-compaction cardiomyopathy, can be caused by a combination of rare, inherited heterozygous missense variants (Gifford et al. 2018). Most recently, Cowan et al. (2018) demonstrated that additional genetic variants underlie dilated cardiomyopathy in 6 families with non-segregating LMNA pedigrees and support multigenic and bilineal inheritance in these families (Cowan et al. 2018). In the light of all these studies and the thorough genetic analysis of the present family, our results provide further evidence of oligogenic pattern of inheritance in complex cardiac disorder such as sudden cardiac death.

It is noteworthy that, clinically, none of the sequenced member of the present family corresponds to a fully recognizable cardiac disease or syndrome. Recent clinical follow-up of the mother (II:2) and her daughter (III:5) did not reveal any clinical manifestations of a definite diagnosis of the initially suspected diseases, namely, Brugada syndrome, arrhythmogenic cardiomyopathy, dilated cardiomyopathy, early repolarization syndrome and CPVT. The phenotypic variability in this family, as well as the anticipated age of sudden 
death among the third generation, are likely caused by the mutational load of all variants in genes involved in different cardiac pathways.

In conclusion, whole exome sequencing of several individuals of the same family and segregation analysis allowed us to identify multiple variants likely responsible for sudden cardiac death.

As calcium is highly required for cardiac muscle excitation-contraction coupling, we hypothesized that calcium mishandling is the most plausible pathophysiological cause of lethal arrhythmias among deceased children.

\section{Acknowledgements}

We would like to thank the family for their collaboration. We gratefully acknowledge Dr. Florence Kyndt and Dr. Juliette Albuisson for their help in SCN5A sequencing. This work was supported by the Tunisian Ministry of Public Health, the Ministry of Higher Education and Scientific Research (LR16IPT05) and Excellence Initiative of Aix-Marseille University A*MIDEX, a French “Investissements d'Avenir” programme (RARE-MED project).

\section{Funding Sources}

This research received no specific grant from any funding agency, commercial or not-forprofit sectors.

Conflicts of interest: The authors declare that they have no conflict of interest.

Ethical approval: All procedures performed in studies involving human participants were in accordance with the ethical standards of the institutional and/or national research 
committee (Registration number: IRB00005445, FWA00010074) and with the 1964 Helsinki declaration and its later amendments or comparable ethical standards.

Informed consent: Written informed consent was obtained from the family members included in this study or their guardians.

\section{Author contributions statement}

Study concept and design: SA, SZ, VD, NL, HJ.

Clinical Investigation of the patient and family members: SC, RM, AZ, LK.

Molecular investigation: $\mathrm{HJ}$.

Analysis and interpretation of data: $\mathrm{HJ}, \mathrm{YB}, \mathrm{HD}, \mathrm{SE}, \mathrm{MN}, \mathrm{SZ}, \mathrm{SA}$.

Supervision: RB, SA, SZ.

Validation: SA, SC, SZ.

Writing - Original Draft Preparation: HJ.

Critical - Review \& Editing: RB, LK, SA, SZ.

\section{References}

Adabag AS, Luepker RV, Roger VL, Gersh BJ (2010) Sudden cardiac death: epidemiology and risk factors. Nat Rev Cardiol 7:216-225. https://doi.org/10.1038/nrcardio.2010.3

Agullo-Pascual E, Cerrone M, Delmar M (2014) Arrhythmogenic cardiomyopathy and Brugada syndrome: diseases of the connexome. FEBS Lett 588:1322-1330. https://doi.org/10.1016/j.febslet.2014.02.008

Arimura T, Matsumoto Y, Okazaki O, et al (2007) Structural analysis of obscurin gene in hypertrophic cardiomyopathy. Biochem Biophys Res Commun 362:281-287. https://doi.org/10.1016/j.bbrc.2007.07.183

Bagnall RD, Das K J, Duflou J, Semsarian C (2014) Exome analysis-based molecular autopsy in cases of sudden unexplained death in the young. Heart Rhythm 11:655-662. https://doi.org/10.1016/j.hrthm.2014.01.017 
Basso C, Carturan E, Pilichou K, et al (2010) Sudden cardiac death with normal heart: Cardiovasc Pathol 19:321-325. https://doi.org/10.1016/j.carpath.2010.02.003

Behr E, Wood DA, Wright M, et al (2003) Cardiological assessment of first-degree relatives in sudden arrhythmic death syndrome. Lancet Lond Engl 362:1457-1459

Behr ER, Dalageorgou C, Christiansen M, et al (2008) Sudden arrhythmic death syndrome: familial evaluation identifies inheritable heart disease in the majority of families. Eur Heart J 29:1670-1680. https://doi.org/10.1093/eurheartj/ehn219

Bezzina CR, Lahrouchi N, Priori SG (2015) Genetics of Sudden Cardiac Death. Circ Res 116:1919-1936. https://doi.org/10.1161/CIRCRESAHA.116.304030

Brion M, Allegue C, Santori M, et al (2012) Sarcomeric gene mutations in sudden infant death syndrome (SIDS). Forensic Sci Int 219:278-281. https://doi.org/10.1016/j.forsciint.2012.01.018

Brion M, Blanco-Verea A, Sobrino B, et al (2014) Next generation sequencing challenges in the analysis of cardiac sudden death due to arrhythmogenic disorders. Electrophoresis 35:3111-3116. https://doi.org/10.1002/elps.201400148

Brion M, Sobrino B, Martinez M, et al (2015) Massive parallel sequencing applied to the molecular autopsy in sudden cardiac death in the young. Forensic Sci Int Genet 18:160-170. https://doi.org/10.1016/j.fsigen.2015.07.010

Cerrone M, Delmar M (2014) Desmosomes and the sodium channel complex: implications for arrhythmogenic cardiomyopathy and Brugada syndrome. Trends Cardiovasc Med 24:184-190. https://doi.org/10.1016/j.tcm.2014.02.001

Christiansen SL, Hertz CL, Ferrero-Miliani L, et al (2016) Genetic investigation of 100 heart genes in sudden unexplained death victims in a forensic setting. Eur J Hum Genet 24:1797-1802. https://doi.org/10.1038/ejhg.2016.118

Coll M, Pérez-Serra A, Mates J, et al (2017) Incomplete Penetrance and Variable Expressivity: Hallmarks in Channelopathies Associated with Sudden Cardiac Death. Biology 7:. https://doi.org/10.3390/biology7010003

Coste B, Mathur J, Schmidt M, et al (2010) Piezo1 and Piezo2 are essential components of distinct mechanically activated cation channels. Science 330:55-60. https://doi.org/10.1126/science. 1193270

Cowan JR, Kinnamon DD, Morales A, et al (2018) Multigenic Disease and Bilineal Inheritance in Dilated Cardiomyopathy Is Illustrated in Nonsegregating LMNA Pedigrees. Circ Genomic Precis Med 11:e002038. https://doi.org/10.1161/CIRCGEN.117.002038

de Villiers CP, van der Merwe L, Crotti L, et al (2014) AKAP9 is a Genetic Modifier of Congenital Long-QT Syndrome Type 1. Circ Cardiovasc Genet 7:599-606. https://doi.org/10.1161/CIRCGENETICS.113.000580 
Delmar M (2012) Desmosome-Ion Channel Interactions and Their Possible Role in Arrhythmogenic Cardiomyopathy. Pediatr Cardiol 33:975-979. https://doi.org/10.1007/s00246-012-0257-0

Devalla HD, Gélinas R, Aburawi EH, et al (2016) TECRL, a new life-threatening inherited arrhythmia gene associated with overlapping clinical features of both LQTS and CPVT. EMBO Mol Med 8:1390-1408. https://doi.org/10.15252/emmm.201505719

Franzini-Armstrong C (2004) Functional implications of RyR-dHPR relationships in skeletal and cardiac muscles. Biol Res 37:507-512

Gifford CA, Ranade SS, Samarakoon R, et al (2018) Oligogenic inheritance of congenital heart disease involving a NKX2-5 modifier. https://doi.org/10.1101/266726

Hata Y, Kinoshita K, Mizumaki K, et al (2016) Postmortem genetic analysis of sudden unexplained death syndrome under 50 years of age: A next-generation sequencing study. Heart Rhythm 13:1544-1551. https://doi.org/10.1016/j.hrthm.2016.03.038

Huang G, Kim JY, Dehoff M, et al (2007) $\mathrm{Ca}^{2+}$ Signaling in Microdomains: Homer1 MEDIATES THE INTERACTION BETWEEN RyR2 AND Cav1.2 TO REGULATE EXCITATION-CONTRACTION COUPLING. J Biol Chem 282:14283-14290. https://doi.org/10.1074/jbc.M611529200

Huang L, Liu C, Tang S, et al (2014) Postmortem genetic screening of SNPs in RyR2 gene in sudden unexplained nocturnal death syndrome in the southern Chinese Han population. Forensic Sci Int 235:14-18. https://doi.org/10.1016/j.forsciint.2013.12.007

Kauferstein S, Kiehne N, Jenewein T, et al (2013) Genetic analysis of sudden unexplained death: A multidisciplinary approach. Forensic Sci Int 229:122-127. https://doi.org/10.1016/j.forsciint.2013.03.050

Lahrouchi N, Raju H, Lodder EM, et al (2017) Utility of Post-Mortem Genetic Testing in Cases of Sudden Arrhythmic Death Syndrome. J Am Coll Cardiol 69:2134-2145. https://doi.org/10.1016/j.jacc.2017.02.046

Li L, Bainbridge MN, Tan Y, et al (2017) A Potential Oligogenic Etiology of Hypertrophic CardiomyopathyNovelty and Significance: A Classic Single-Gene Disorder. Circ Res 120:1084-1090. https://doi.org/10.1161/CIRCRESAHA.116.310559

Lopes LR, Murphy C, Syrris P, et al (2015) Use of high-throughput targeted exomesequencing to screen for copy number variation in hypertrophic cardiomyopathy. Eur $\mathrm{J}$ Med Genet 58:611-616. https://doi.org/10.1016/j.ejmg.2015.10.001

Maron BJ (2003) Sudden death in young athletes. N Engl J Med 349:1064-1075. https://doi.org/10.1056/NEJMra022783

Marston S (2017a) Obscurin variants and inherited cardiomyopathies. Biophys Rev. https://doi.org/10.1007/s12551-017-0264-8 
Marston S (2017b) Obscurin variants and inherited cardiomyopathies. Biophys Rev 9:239_ 243. https://doi.org/10.1007/s12551-017-0264-8

Marston S, Montgiraud C, Munster AB, et al (2015) OBSCN Mutations Associated with Dilated Cardiomyopathy and Haploinsufficiency. PLOS ONE 10:e0138568. https://doi.org/10.1371/journal.pone.0138568

Neubauer J, Lecca MR, Russo G, et al (2017) Post-mortem whole-exome analysis in a large sudden infant death syndrome cohort with a focus on cardiovascular and metabolic genetic diseases. Eur J Hum Genet 25:404-409. https://doi.org/10.1038/ejhg.2016.199

Neubauer J, Lecca MR, Russo G, et al (2018) Exome analysis in 34 sudden unexplained death (SUD) victims mainly identified variants in channelopathy-associated genes. Int J Legal Med. https://doi.org/10.1007/s00414-018-1775-y

Noorman M, Hakim S, Kessler E, et al (2013) Remodeling of the cardiac sodium channel, connexin43, and plakoglobin at the intercalated disk in patients with arrhythmogenic cardiomyopathy. Heart Rhythm Off J Heart Rhythm Soc 10:412-419. https://doi.org/10.1016/j.hrthm.2012.11.018

Plagnol V, Curtis J, Epstein M, et al (2012) A robust model for read count data in exome sequencing experiments and implications for copy number variant calling. Bioinforma Oxf Engl 28:2747-2754. https://doi.org/10.1093/bioinformatics/bts526

Priori SG, Borggrefe M, Camm AJ, et al (1992) Unexplained cardiac arrest. The need for a prospective registry. Eur Heart J 13:1445-1446

Priori SG, Wilde AA, Horie M, et al (2013) HRS/EHRA/APHRS expert consensus statement on the diagnosis and management of patients with inherited primary arrhythmia syndromes: document endorsed by HRS, EHRA, and APHRS in May 2013 and by ACCF, AHA, PACES, and AEPC in June 2013. Heart Rhythm 10:1932-1963. https://doi.org/10.1016/j.hrthm.2013.05.014

Rode B, Shi J, Endesh N, et al (2017) Piezol channels sense whole body physical activity to reset cardiovascular homeostasis and enhance performance. Nat Commun 8:350. https://doi.org/10.1038/s41467-017-00429-3

Sarquella-Brugada G, Campuzano O, Cesar S, et al (2016) Sudden infant death syndrome caused by cardiac arrhythmias: only a matter of genes encoding ion channels? Int J Legal Med 130:415-420. https://doi.org/10.1007/s00414-016-1330-7

Sutphin BS, Boczek NJ, Barajas-Martínez H, et al (2016) Molecular and Functional Characterization of Rare CACNA1C Variants in Sudden Unexplained Death in the Young. Congenit Heart Dis 11:683-692. https://doi.org/10.1111/chd.12371

Tan R, Wang Y, Kleinstein SE, et al (2014) An evaluation of copy number variation detection tools from whole-exome sequencing data. Hum Mutat 35:899-907. https://doi.org/10.1002/humu.22537 
van den Hoogenhof MMG, Beqqali A, Amin AS, et al (2018) RBM20 Mutations Induce an Arrhythmogenic Dilated Cardiomyopathy Related to Disturbed Calcium Handling. Circulation. https://doi.org/10.1161/CIRCULATIONAHA.117.031947

van der Werf C, van Langen IM, Wilde AAM (2010) Sudden death in the young: what do we know about it and how to prevent? Circ Arrhythm Electrophysiol 3:96-104. https://doi.org/10.1161/CIRCEP.109.877142

Wells QS, Becker JR, Su YR, et al (2013) Whole exome sequencing identifies a causal RBM20 mutation in a large pedigree with familial dilated cardiomyopathy. Circ Cardiovasc Genet 6:317-326. https://doi.org/10.1161/CIRCGENETICS.113.000011

Wilhelm M, Bolliger SA, Bartsch C, et al (2015) Sudden cardiac death in forensic medicine Swiss recommendations for a multidisciplinary approach. Swiss Med Wkly 145:w14129. https://doi.org/10.4414/smw.2015.14129

Winkel BG, Holst AG, Theilade J, et al (2011) Nationwide study of sudden cardiac death in persons aged 1-35 years. Eur Heart J 32:983-990. https://doi.org/10.1093/eurheartj/ehq428

Wong T-Y, Juang W-C, Tsai C-T, et al (2018) Mechanical Stretching Simulates Cardiac Physiology and Pathology through Mechanosensor Piezo1. J Clin Med 7:. https://doi.org/10.3390/jcm7110410

Xie L, Hou C, Jiang X, et al (2019) A compound heterozygosity of Tecrl gene confirmed in a catecholaminergic polymorphic ventricular tachycardia family. Eur J Med Genet 62:103631. https://doi.org/10.1016/j.ejmg.2019.01.018

Table 1: List of variants identified in this study.

Table 2: Prediction of variant deleteriousness based on CADD-scores. 


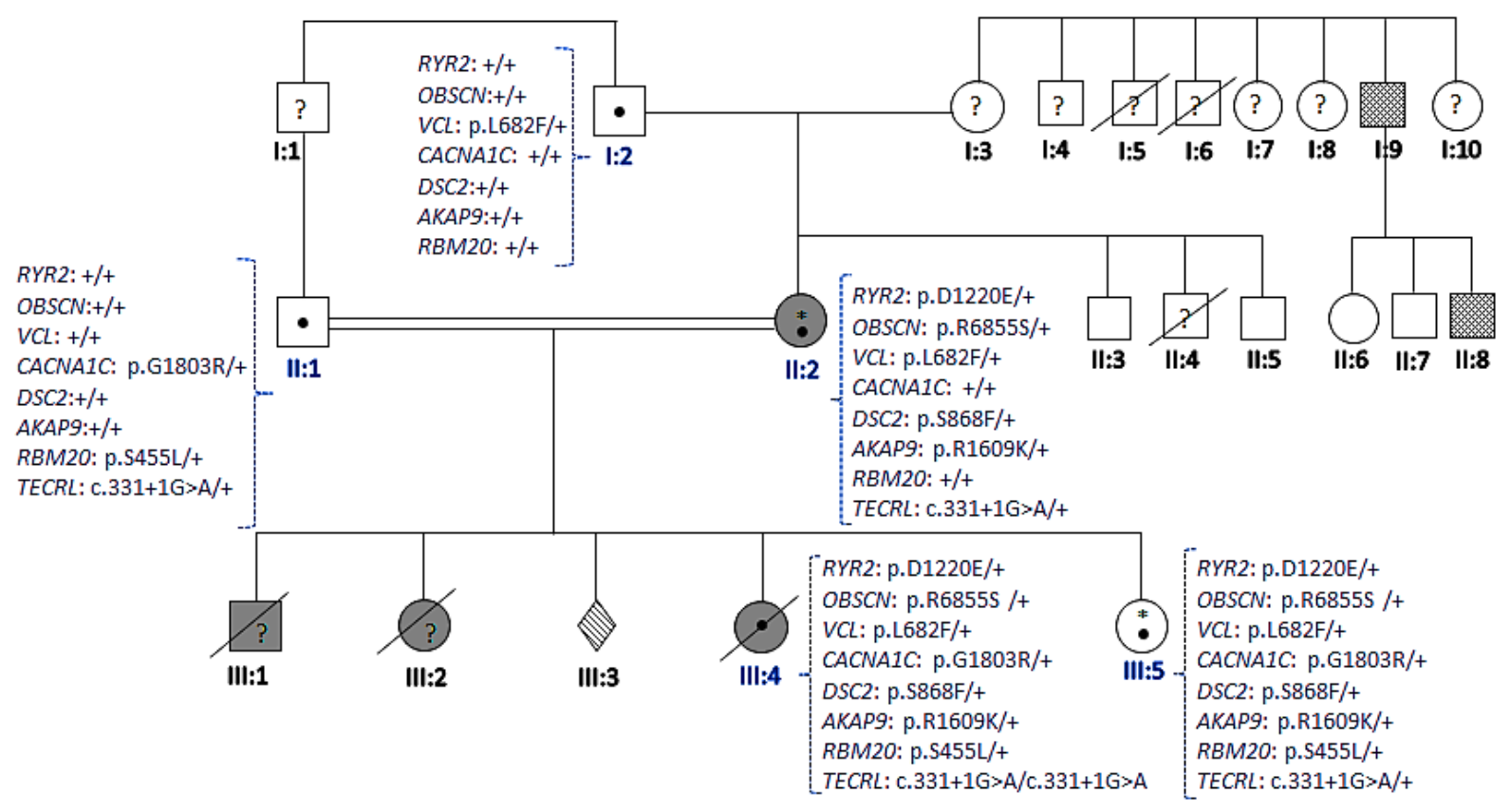

Fig. 1: Pedigree of the family. The white symbols represent healthy members; the filled gray symbols represent affected members. The crossed symbols represent deceased members. The members highlighted by asterisks received an ICD. The individuals highlighted by black dot were genetically and clinically evaluated and those by question mark were genetically and clinically not evaluated. The autopsied fetus is marked by the diamond symbol. The candidate variants are listed beside each member. Plus sign indicates the wild type allele. 


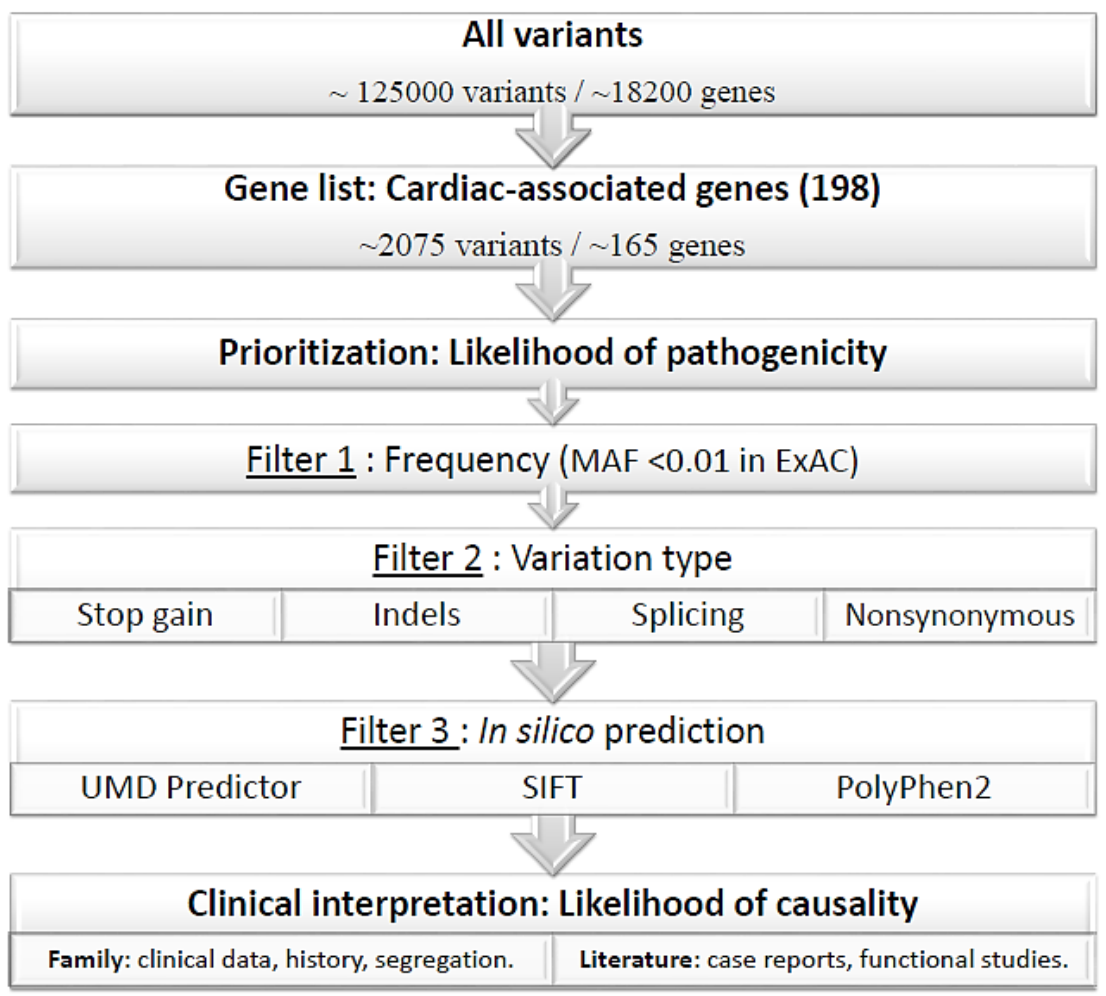

Fig.2: Filtering strategy after automatic variant calling and annotation.

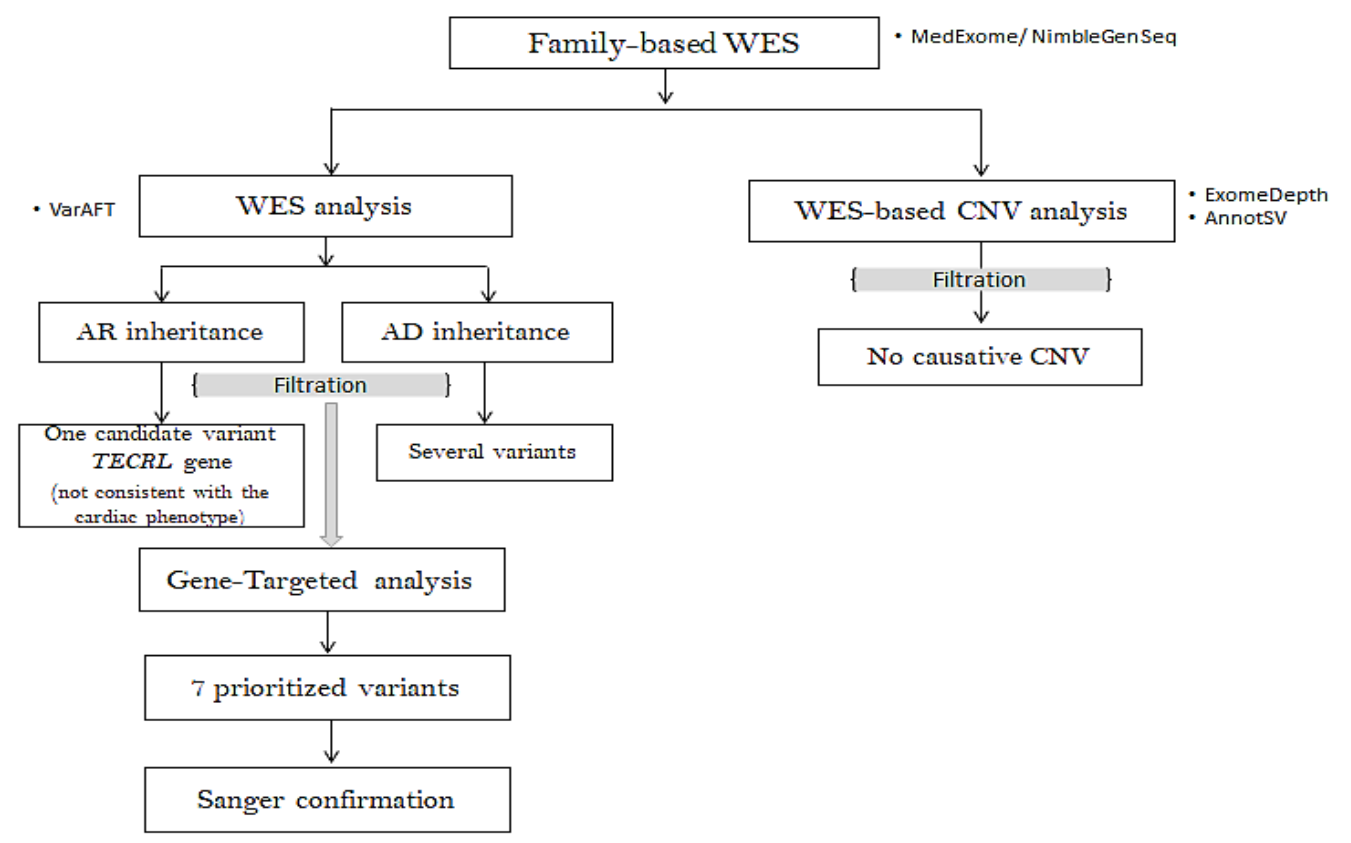

Fig.3: Genetic study design flowchart. 